\title{
Effects of Shikunshito-Kamiho on Fecal Enzymes and Formation of Aberrant Crypt Foci Induced by 1,2-Dimethylhydrazine
}

\author{
Bong-Ha Yoo, ${ }^{a}$ Beom-Han LeE, ${ }^{a}$ Jin-Sung KIm, ${ }^{a}$ Nam-Jae KIm, ${ }^{c}$ Sung-Hoon KIM, ${ }^{*, b}$ and \\ $\mathrm{Ki}-$ Won $\mathrm{RYU}^{b}$ \\ Department of Internal Medicine, Oriental Medical College, Kyunghee University, ${ }^{a} 1$ Hoegi-dong, Dongdaemun-gu, Seoul \\ 130-701, Korea, Department of Oncology, Graduate School of East-West Medical Science, ${ }^{b} 1$ Seochunri, Kiheungeup, \\ Yongin 449-701, Korea, and Department of Oriental Pharmacy, Oriental Medical Hospital, Kyunghee University, ${ }^{c} 1$ \\ Hoegi-dong, Dongdaemun-gu, Seoul 130-701, Korea. Received October 10, 2000; accepted February 8, 2001
}

\begin{abstract}
Shikunshito-Kamiho (SKTK) is a traditional Chinese medicine composed of eight crude drugs (Ginseng Radix, Hoelen, Atractylodis Rhizoma, Glycyrrhizae Radix, Prunellae Spica, Ostreae Testa, Laminaria Thallus, Sargassum). We investigated the effects of SKTK on pH, ammonia, fecal enzymes such as $\boldsymbol{\beta}$-glucuronidase, tryptophanase, urease, and formation aberrant crypt foci in the colon carcinogenesis model induced by 1,2-dimethylhydrazine (DMH). Water extract of SKTK was administered orally for 5 weeks to DMH-treated mice as $0.5 \%$ and $1.5 \%$ of the diet. $\beta$-Glucuronidase, $\mathrm{pH}$ and tryptophanase were significantly inhibited after treatment of $0.5 \%$ and $1.5 \%$ SKTK, while urease was significantly reduced only during and after treatment of $1.5 \%$ SKTK as compared with control data. However, the ammonia concentration wasn't different in SKTK treated groups from control group. The incidence number of aberrant crypts foci (ACF) and aberrant crypts/focus in colon was significantly decreased by $0.5 \%$ and $1.5 \%$ SKTK mixed diets compared with that in rats treated with DMH alone. These results suggest that SKTK exterts anticarcinogenic activity on experimental murine colorectal cancer.
\end{abstract}

Key words SKTK (Shikunshito-Kamiho); 1,2-dimethylhydrazine; $\beta$-glucuronidase; tryptophanase; urease; aberrant crypts foci

Human colorectal cancers are mainly caused by exposure to food mutagens and carcinogens. ${ }^{1)}$ 1,2-Dimethylhydrazine (1,2-DMH) is a typical colon carcinogen which conjugates with glucuronic acid in liver and is excreted in intestine via the bile. ${ }^{2)}$ The glucuronide conjugates hydrolysed by bacterial $\beta$-glucosidase are known to increase the activities of fecal enzymes such as $\beta$-glucuronidase, tryptophanase and urease in metabolic pathways. ${ }^{3)}$

Aberrant crypt foci (ACF) in colonic mucosa were first observed in the carcinogen treated rodent $\operatorname{colon}^{8)}$ and were also known to be putative preneoplastic lesions in colon of both rodents and humans. ${ }^{4)}$ Induction of K-ras mutation and increased expression of ras and c-fos oncogene products in $\mathrm{ACF}$ have been reported ${ }^{5)}$ suggesting ACF in the rodent colon can be used as a good biomarker in colon carcinogenesis.

Chemoprevention is an alternative approach to decrease the carcinogenic effect. Natural chemopreventive agents have inhibitory effects on carcinogenesis. ${ }^{6}$ ) Medicinal plants are also candidates for cancer chemoprevention. ShikunshitoKamiho (SKTK) is an oriental prescription consisting of eight herbs used for the treatment of colorectal cancer in oriental medicine. ${ }^{7}$ However, there wasn't yet any experimental data for SKTK which includes three kinds of sea weeds.

Thus, in the present study, we investigated the anticarcinogenic effects of SKTK on 1,2-DMH-induced colorectal cancer in vivo by evaluating $\mathrm{pH}$, ammonia, fecal enzymes such as $\beta$-glucuronidase, tryptophanase and urease, and also ACF formation in the murine colon.

\section{MATERIALS AND METHODS}

Animals SPF male ICR mice (18-24 g body weight) were provided by the Laboratory Animal Center, Korea Research Institute of Chemistry and Technology. Mice were in- dividually housed in polycarbonate cages and maintained in a temperature $\left(23 \pm 2{ }^{\circ} \mathrm{C}\right)$ and humidity $(50 \pm 10 \%)$ controlled animal facility with a daily $12: 12 \mathrm{~h}$ light-dark photoperiod. After a 2 week acclimatization period of consuming standard rat chow, the rats were used for the experiment.

Materials Medicinal herbs of Shikunshito-Kamiho (SKTK) were purchased from Koryo Company of Traditional Crude Drugs (Seoul, Korea) and kindly authenticated by Dr. Deok-Kyun Ahn, Professor of Department of Herbology, Oriental Medical College, Kyunghee University. SKTK $(40.0 \mathrm{~g})$ consists of eight crude drugs (Panax ginseng C. A. Meyer, Astractylodes macrocephala Koidzumi, Poria cocos WolfF, Glycyrrhiza ularensis Fisch, Prunella vulgaris var. lilacina NAKAI, Sargassum pallidum (Turn.) C. Ag., Laminaria japonica ARESCH and Ostrea gigas ThUBERg). The composition of SKTK, botanical origins, crude drugs, voucher number, harvesting, place and dose of each herb are shown in Table 1. Voucher specimens were deposited in the herbarium of the Museum of Materia Medica, Oriental Medical College, Kyunghee University, Seoul, Korea. The qualities of medicinal herbs were controlled by Korean Pharmacopeia VII which regulates the botanical origin, the crude drug, loss of drying, total ash, test of foreign matter, acid insoluble ash, extract content, essential oil content and microscopic examination. ${ }^{14)}$

Preparation of SKTK Extract One and two tenths kilograms of SKTK was mixed and boiled with regular water. The boiled solution was concentrated with rotary evaporator (Model NE-1, Japan) and dried with freeze dryer (Model FD1, Japan) to obtain $195 \mathrm{~g}$ of SKTK extract. The yield of water extract of SKTK was $16.25 \%(\mathrm{w} / \mathrm{w})$ in terms of the dried medicinal herbs. Usually, the daily dose of SKTK extract in the treatment of human is $19.5 \mathrm{~g}$ three times a day. Thus, the daily dose ratio for a mouse was determined as $0.5 \%$ and $1.5 \%$ of regular diet considering daily food intake (approxi- 
mately $4 \mathrm{~g}$ ) and drug susceptibility.

Identification of Major Chemical Components in SKTK by HPLC Profile Analysis Chemical components such as glycyrrhizin, ginsenoside $\mathrm{Rb} 1, \mathrm{Rg} 1$ and ursolic acid were identified in SKTK by HPLC analysis under the following conditions. Glycyrrhizin: Extraction solvent, 70\% ethanol; column, Nucleosil 120-5 C18 $(4.6 \times 250 \mathrm{~mm})$, mobile phase, $\mathrm{H}_{2} \mathrm{O}: \mathrm{CH}_{3} \mathrm{CN}$ : tetrabutylammonium bromide $(620 \mathrm{ml}: 380 \mathrm{ml}: 1.5 \mathrm{~g})$; flow rate, $1.5 \mathrm{ml} / \mathrm{min}$; detector, Waters $^{\mathrm{TM}} 486(254 \mathrm{~nm})$; room temperature, $11^{\prime} 18^{\prime \prime}$. Ginsenoside $\mathrm{Rb} 1$ : Extraction solvent, $70 \%$ methanol; column, $\mu$-Bondapak $\mathrm{C} 18(4.6 \times 250 \mathrm{~mm})$, mobile phase, $\mathrm{H}_{2} \mathrm{O}: \mathrm{CH}_{3} \mathrm{CN}$ (71:29); flow rate, $2.0 \mathrm{ml} / \mathrm{min}$; detector, Waters ${ }^{\mathrm{TM}} 486$ $(203 \mathrm{~nm})$; room temperature, 13'54". Ginsenoside Rg1 : Extraction solvent, $70 \%$ methanol; column, $\mu$-Bondapak $\mathrm{C} 18$ (4.6×250 mm), mobile phase, $\mathrm{H}_{2} \mathrm{O}: \mathrm{CH}_{3} \mathrm{CN}$ (81:19); flow rate, $2.0 \mathrm{ml} / \mathrm{min}$; detector, Waters ${ }^{\mathrm{TM}} 486(203 \mathrm{~nm})$; room temperature, 13'54". Ursolic acid: Extraction solvent, methanol; column, $\mu$-Bondapak C18 $(4.6 \times 250 \mathrm{~mm})$, mobile phase, $\mathrm{H}_{2} \mathrm{O}: \mathrm{CH}_{3} \mathrm{CN}: \mathrm{MeOH}(6: 12: 5)$; flow rate, $1.5 \mathrm{ml} / \mathrm{min}$; detector, Waters ${ }^{\mathrm{TM}} 486(213 \mathrm{~nm})$; room temperature, $\left.5^{\prime} 5^{\prime \prime} .{ }^{15}\right)$

Induction of Colorectal Cancer by 1,2-DMH and Treatment Male mice housed in a plastic cage were acclimated for $3 \mathrm{~d}$ before starting the experiment. Water and diet were administered ad libitum. At the age of 5 weeks, 120 mice were divided into 4 groups such as normal group, control group, Sample I group and Sample II group. To induce colorectal cancer 1,2-DMH was injected subcutaneously into mice of control, Sample I and Sample II groups once a week for 10 weeks, while saline was injected into the normal group. Sample I and Sample II groups were fed with the usual powdered diets containing the $0.5 \%$ and $1.5 \%$ water extracts of SKTK respectively for 5 weeks after last injection of 1,2-DMH.

Assay of Fecal Enzyme Activities The activities of $\beta$ glucuronidase, tryptophanase and urease as fecal enzymes were evaluated from feces collected 2 weeks before, during and 2 weeks after SKTK treatment. All procedures were performed at $4{ }^{\circ} \mathrm{C}$. An aliquot of $2 \mathrm{~g}$ of homogenized fecal sample was taken in liquid nitrogen and dismemberment was done in Teflon plots for $8 \mathrm{~min}$ at an amplitude of $1 \mathrm{~cm}$ in a microdismemberator. Approximately $2 \mathrm{~g}$ of dismembered samples were added to $10 \mathrm{ml}$ of phosphate-buffered saline (PBS, pH 6) and mixed well for $1 \mathrm{~min}$, and the enzyme extract was used for assaying the enzyme activities of $\beta$-glucuronidase. For measuring tryptophanase activity, $1 \mathrm{ml}$ of fecal suspension was taken in $1 \mathrm{ml}$ of $0.05 \mathrm{~m}$ PBS (pH 6.5) buffer and to this was added $2 \mathrm{ml}$ of cold acetone, mixed well, centrifuged at $2000 \mathrm{~g}$ for $15 \mathrm{~min}$ at $4{ }^{\circ} \mathrm{C}$ and the supernatant was used for the assaying the activity at $540 \mathrm{~nm}$. Urease activity was measured using a kit obtained from Boehringer Mannheim GmbH (Cat. No. 54296). All the enzyme activities were evaluated spectrophotometrically.

Determination of ACF (Aberrant Crypt Foci) ACF in the rat colon were scored as described by Bird. ${ }^{8}$ After oral treatment of SKTK for 5 weeks after subcutaneous injection of DMH for 10 weeks, the colons of sacrificed mice were removed and expanded with $10 \%$ formaldehyde in PBS, $\mathrm{pH}$ 7.4. The colons were kept on ice for $15 \mathrm{~min}$. Then they were cut into three parts, the rectum, sigmoid and descending colon and cut open along the longitudinal median axis. Then pieces of the colon were placed flat between two pieces of filter paper, fixed in $10 \%$ formalin-PBS solution for at least $16 \mathrm{~h}$, and stained with $0.2 \%$ methylene blue for $1-2 \mathrm{~min}$. Foci of aberrant crypts were scored under a light microscope at a magnification of $\times 100$.

Statistical Analysis The data were analyzed statistically by analysis of variance.

\section{RESULTS AND DISCUSSION}

Shikunshito-Kamiho (SKTK) is a traditional prescription based on Shikunshito by adding four herbs (Table 1). Shikunshito has been frequently used to supplement Qi, vital energy, consisting of four herbs such as Panax ginseng C. A. Meyer, Astractylodes macrocephala KoIDzumi, Poria cocos Wolff, Glycyrrhiza ularensis Fisch. ${ }^{9)}$ Oriental prescriptions based on Shikunshito also were clinically applied to cancer treatment. ${ }^{10)}$ Four herbs added to SKTK were Ostreae Concha, Laminaria Thallus, Sargassum and Prunellae Spica to reinforce the anticancer activity of Shikunshito. Of those three herbs were sea weeds having herbological effect to soften callus or hard mass and abundant vitamin $\mathrm{C}$ and Prunellae Spica was reported to have antitumor activity against lymphoma. ${ }^{11)}$

In order to confirm the presenece of Panax ginseng, Prunellae Spica, and Glycyrrhiza ularensis in the prescription of SKTK, the principal components of the herbs were quantitatively analyzed by HPLC. The contents of ginsenoside Rb1 and Rg1, the principal components of Panax ginseng, glycyrrhizin, that of Glycyrrhiza ularensis, and ursolic acid, that of Prunellae Spica were determined to be 0.111 , $0.097,1.51$, and $0.009 \%$, respectively (Fig. 1).

We evaluated the anticarcinogenic effects of SKTK on 1,2$\mathrm{DMH}$-induced colorectal cancer by measuring $\mathrm{pH}$, ammonia, fecal enzymes such as $\beta$-glucuronidase, tryptophanase and

Table 1. The Composition of SKTK, Botanical Origins, Crude Drugs, Voucher Number, Harvesting, Place and Dose of Each Herb

\begin{tabular}{|c|c|c|c|c|c|}
\hline Botanical origin $^{a}$ ) & Crude drugs & Voucher specimen number & Harvesting & Place & Dose ratio $(\mathrm{g})$ \\
\hline Panax ginseng C. A. MEYER & Ginseng Radix & GSM9901 & Autumn & Korea & 5.0 \\
\hline Poria cocos WolfF & Hoelen & GSM9902 & Autumn & Korea & 5.0 \\
\hline Astractylodes macrocephala KoIDzUMI & Atractylodis Rhizoma & GSM9903 & Autumn & Korea & 5.0 \\
\hline Glycyrrhiza ularensis Fisch & Glycyrrhizae Rhizoma & GSM9904 & Autumn & China & 5.0 \\
\hline Prunella vulgaris var. lilacina NAKAI & Prunellae Spica & GSM9905 & Summer & Korea & 5.0 \\
\hline Ostrea gigas THUNBERG & Ostreae Concha & GSM9906 & Four seasons & Korea & 5.0 \\
\hline Laminaria japonica ARESCH. & Laminaria Thallus & GSM9907 & Summer to winter & Korea & 5.0 \\
\hline Sargassum pallidum (TurN.) C. Ag. & Sargassum & GSM9908 & Summer to winter & Korea & 5.0 \\
\hline
\end{tabular}

a) The botanical origins are based on Korean Pharmacopenia VII. b) These drugs were used in SKTK in this study. 

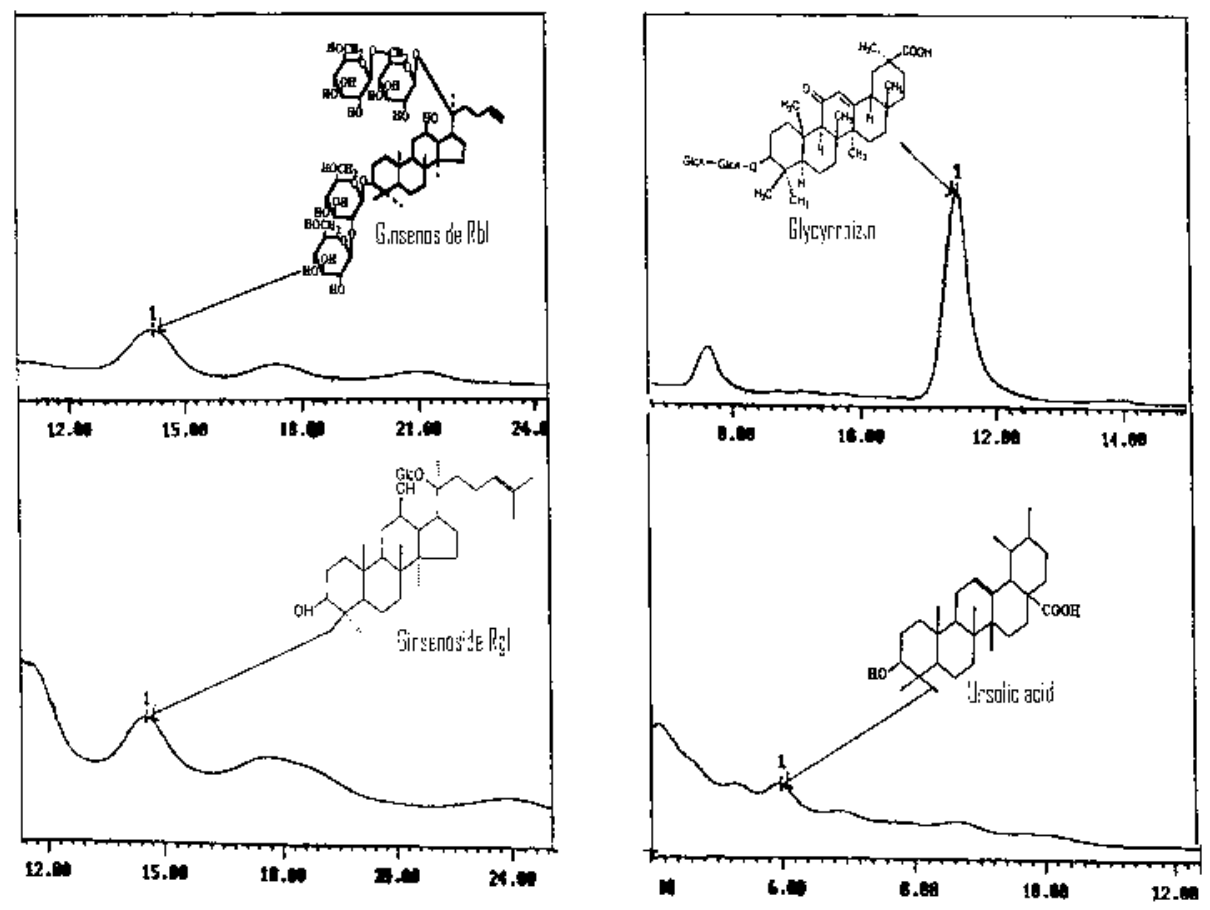

Fig. 1. HPLC Profile of Major Chemical Components of SKTK

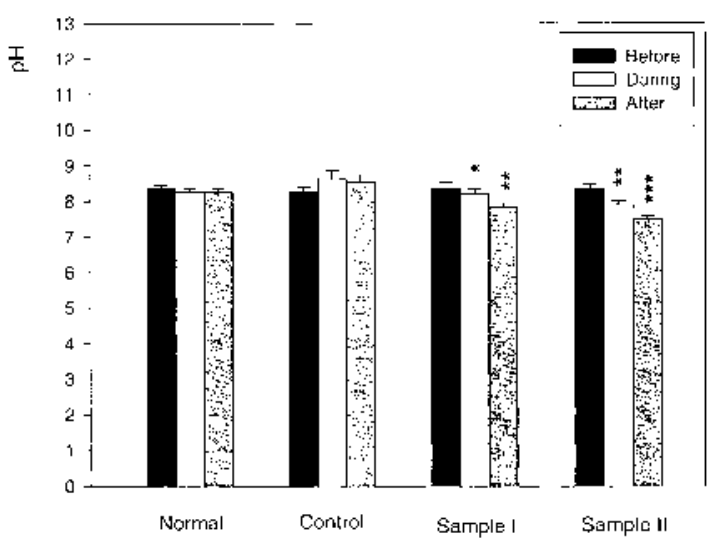

Fig. 2. Effects of SKTK on $\mathrm{pH}$ in the Intestines of Mice

Mice were divided into four groups. Normal and Control groups were fed with the usual powdered laboratory diet. Sample I group was fed with the usual powdered laboratory diet containing the $0.5 \%$ water extract of SKTK. Sample II group was fed with the usual powdered laboratory diet containing the $1.5 \%$ water extract of SKTK. Control, Sample I and Sample II groups were respectively given weekly subcutaneous (s.c.) injection of 1,2-DMH for 10 weeks. All animals were fed for 5 weeks after last injection and then mice were examined by autopsy. Before: before treatment, During: during treatment, After: after treatment. Statistically significant compared to control data $(* p<0.05, * * p<0.01, * * * p<0.001)$.

urease, and also ACF formation in the murine colon. $\mathrm{pH}$ was significantly lowered during and after treatment of Sample I and Sample II groups to 7.85 and 7.5, respectively as compared with control group, $\mathrm{pH} 8.6$ (Fig. 2). $\beta$-Glucuronidase was reported to be closely related to colorectal cancer formation. ${ }^{3)}$ It was significantly suppressed after treatment of Sample I and during and after treatment of Sample II groups to $14.9 \%$ and $21.3 \%$ of control data (Fig. 3). Tryptophanase was reported to resolve tryptophan into indole, ammonia and pyruvic acid, causes of bladder and intestine cancers. ${ }^{12)}$ Tryptophanase was significantly reduced to normal range only after treatment of Sample II, while it wasn't statistically different between control and Sample groups during treatment

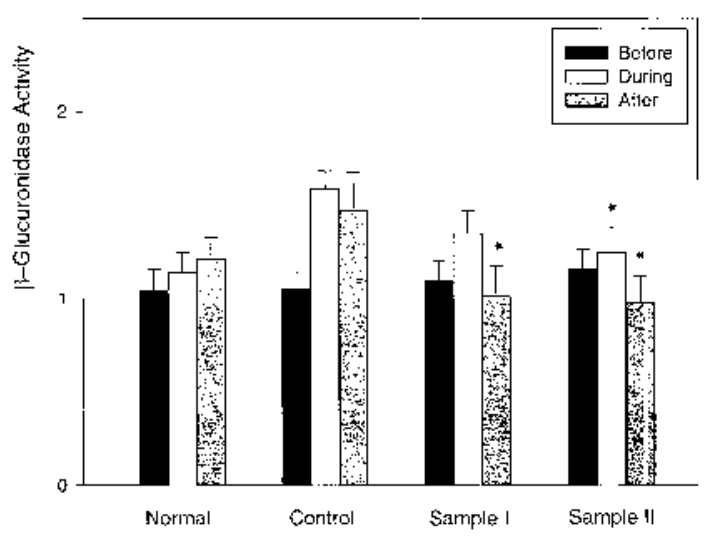

Fig. 3. Effects of SKTK on $\beta$-Glucuronidase Activities Produced by Intestinal Bacteria in Mice

Mice were divided into four groups. Normal and Control groups were fed with the usual powdered laboratory diet. Sample I group was fed with the usual powdered laboratory diet containing the $0.5 \%$ water extract of SKTK. Sample II group was fed with the usual powdered laboratory diet containing the $1.5 \%$ water extract of SKTK. Control, Sample I and Sample II groups were respectively given weekly subcutaneous (s.c.) injection of 1,2-DMH for 10 weeks. All animals were fed for 5 weeks after last injection and then mice were examined by autopsy. Before: before treatment, During: during treatment, After: after treatment. Statistically significant compared to control data $(* p<0.05, * * p<0.01, * * p<0.001)$.

(Fig. 4). Urease is an enzyme that produces ammonia and carbon dioxide, a chief cause of hepatic coma. ${ }^{13)}$ SKTK exterted an inhibitory effect on urease up to $35 \%$ of control at $1 \%$ concentration in vitro, and also significantly lowered urease only during and after treatment of Sample II as compared with control data in vivo (Fig. 5). However, ammonia wasn't different between thae sample groups and control group suggesting produced ammonia was absorbed in intestines or consumed by other bacteria (Fig. 6).

The number of $\mathrm{ACF} /$ colon was significantly decreased to 6.9 and 6.4 , respectively by $0.5 \%$ and $1.5 \%$ SKTK mixed diets while that in control group was significantly increased 


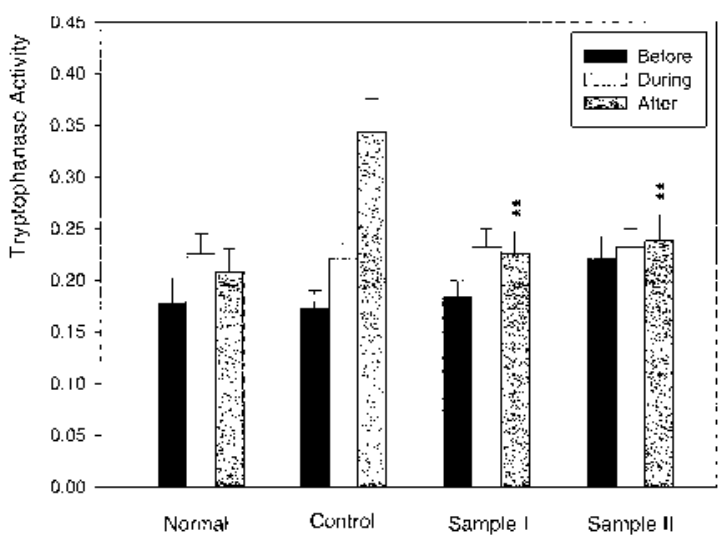

Fig. 4. Effects of SKTK on Tryptophanase Activity Produced by Intestinal Bacteria in Mice

Mice were divided into four groups. Normal and Control groups were fed with the usual powdered laboratory diet. Sample I group was fed with the usual powdered laboratory diet containing the $0.5 \%$ water extract of SKTK. Sample II group was fed with the usual powdered laboratory diet containing the $1.5 \%$ water extract of SKTK. Control, Sample I and Sample II groups were respectively given weekly subcutaneous (s.c.) injection of 1,2-DMH for 10 weeks. All animals were fed for 5 weeks after last injection and then mice were examined by autopsy. Before: before treatment, During: during treatment, After: after treatment. Statistically significant compared to control data $(* p<0.05, * * p<0.01, * * * p<0.001)$.

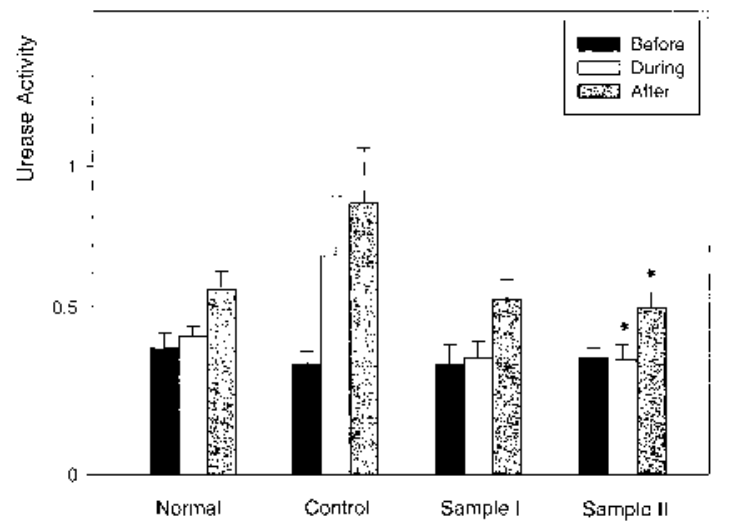

Fig. 5. Effects of SKTK on the Urease Activity Produced by Intestinal Bacteria in Mice

Mice were divided into four groups. Normal and Control groups were fed with the usual powdered laboratory diet. Sample I group was fed with the usual powdered laboratory diet containing the $0.5 \%$ water extract of SKTK. Sample II group was fed with the usual powdered laboratory diet containing the $1.5 \%$ water extract of SKTK. Control, Sample I and Sample II groups were respectively given weekly subcutaneous (s.c.) injection of 1,2-DMH for 10 weeks. All animals were fed for 5 weeks after last injection and then mice were examined by autopsy. Before: before treatment, During: during treatment, After: after treatment. Statistically significant compared to control data $(* p<0.05, * * p<0.01, * * * p<0.001)$.

to 21.6 (Table 2, Figs. 7, 8). Similarly, the formation of aberrant crypts per focus was significantly inhibited compared with control data $(p<0.05)$. ACF also was chiefly distributed in rectum by SKTK treatment rather than in sigmoid and descending colons by DMH alone. However, it was difficult to recognize the dose dependent effect, though the anticarcinogenic effect was significantly exhibited in both sample groups, suggesting toxicological study and another animal study at lower doses. These results suggest that SKTK exterts anticarcinogenic activity on experimental murine colorectal cancer.

Acknowledgement This study was in part supported by

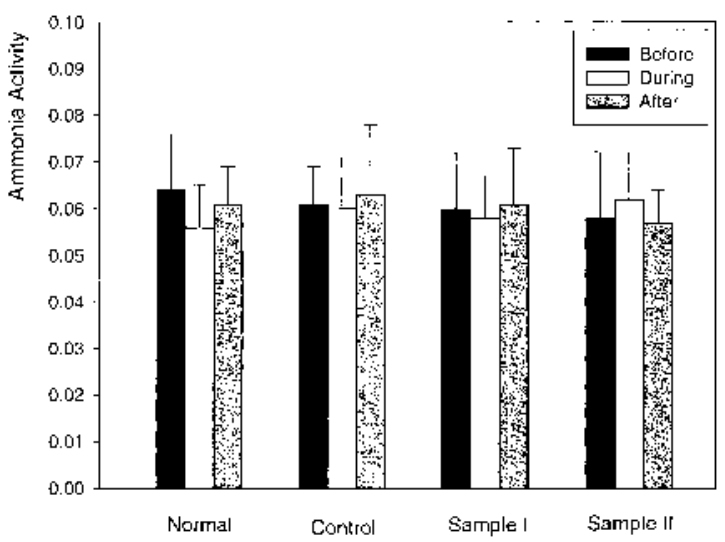

Fig. 6. Effects of SKTK on Ammonia Produced by Intestinal Bacteria in Mice

Mice were divided into four groups. Normal and Control groups were fed with the usual powdered laboratory diet. Sample I group was fed with the usual powdered laboratory diet containing the $0.5 \%$ water extract of SKTK. Sample II group was fed with the usual powdered laboratory diet containing the $1.5 \%$ water extract of SKTK. Control, Sample I and Sample II groups were respectively given weekly subcutaneous (s.c.) injection of 1,2-DMH for 10 weeks. All animals were fed for 5 weeks after last injection and then mice were examined by autopsy. Before: before treatment, During: during treatment, After: after treatment. Statistically significant compared to control data $(* p<0.05, * * p<0.01, * * * p<0.001)$.

Table 2. Effects of SKTK on the Formation of Aberrant Foci (ACF) and Aberrant Crypts (AC) in Murine Colons Induced by 1,2 Dimethylhydrazine

\begin{tabular}{lccccc}
\hline \hline Group & Incidence & $\begin{array}{c}\text { Number } \\
\text { of } \\
\text { ACF/colon }\end{array}$ & $\begin{array}{c}\text { Number } \\
\text { of } \\
\text { AC/Focus }\end{array}$ & R & Sistribution (\%) \\
\cline { 5 - 6 } Normal & $0 / 10$ & 0 & 0 & & \\
Control (DMH) & $10 / 10$ & $21.6 \pm 3.9^{\#}$ & $2.9 \pm 0.3^{\#}$ & 29 & 71 \\
Sample I & $9 / 10$ & $6.9 \pm 1.8^{*}$ & $2.0 \pm 0.1^{*}$ & 41 & 59 \\
Sample II & $8 / 10$ & $6.4 \pm 1.3^{*}$ & $1.9 \pm 0.1^{*}$ & 50 & 50 \\
\hline
\end{tabular}

R: Rectum, S\&D; Sigmoid and colon. \#: Statistically significant compared with normal data $(p<0.05)$. $*$ Statistically significant compared with normal data $(p<0.05)$.

Brain Korea 21 Project and Research Fund of Department of Health and Welfare (HMP99-O-11-0011-B).

\section{REFERENCES}

1) Sugumura T., Sato S., Cancer Res., 43 (Suppl.), 2415s-2421s (1983).

2) Weisburger J. H., Cancer Res., 28, 60-70 (1971).

3) Celika C., Lewis D. A., Mittleman A., J. Surgical Oncology, 24, $209-$ 211 (1983).

4) Tudek B., Bird R. P., Bruce W. R., Cancer Res., 49, 1236-1240 (1989).

5) Stopera S. A., Bird R. P., Carcinogenesis, 13, 573-578 (1992).

6) Wattenberg L. W., Cancer Res., 45, 1-8 (1985).

7) Guoyong, J. Chinese Med., 34, 481-483 (1993).

8) Bird R. P., Cancer Lett., 93, 55-71 (1995).

9) Jun H., "Dongyibaojian,” Namsan Press, 1985, p. 144.

10) Fan Q., Jeolkang Chinese Med. Bull., 6, 22-23 (1983).

11) Kee C. H., "The Pharmacology of Chinese Herbs," CRC Press, 1993, pp. $271-272$.

12) Chung K. T., Fulk G. E., Slein M. W., J. Natl. Cancer. Inst., 54, 1073-1078 (1975).

13) Park J. B., Imamura L., Kobashi K., Itoh H., Miyazaki T., Horisaki T., Biol. Pharm. Bull., 18, 208-213 (1995).

14) Korean Department of Health and Welfare, "Korean Pharmacopeia VII," 1997, pp. 10-16, 708.

15) Won D. H., "Component Analysis of Frequently Used Herbs," 1991, pp. $292-306$. 


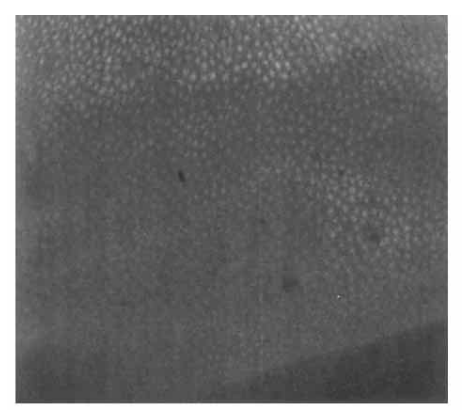

A

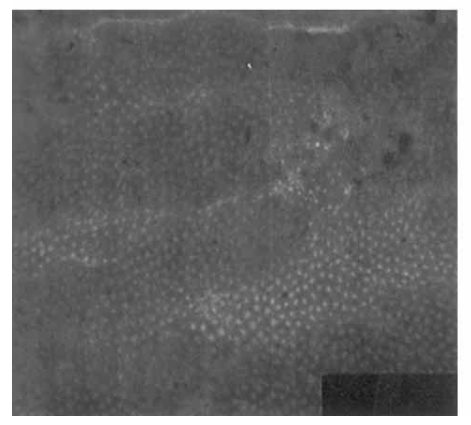

$\mathrm{C}$

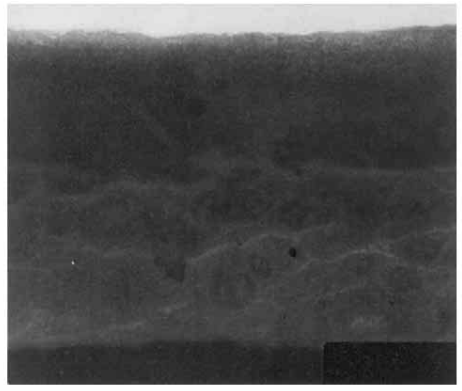

B

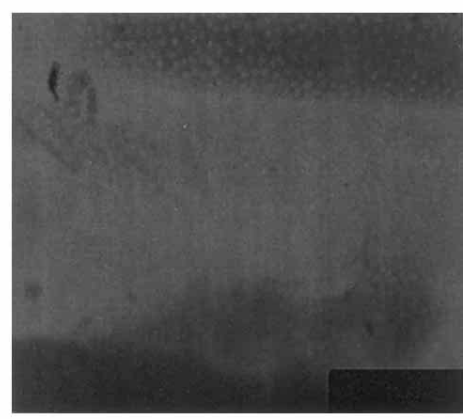

$\mathrm{D}$

Fig. 7. Effect of SKTK on the Formation of Aberrant Foci and Crypts in Murine Colon Induced by 1,2-Dimethylhydrazine A, rectum of normal group; B, rectum of control group; C, rectum of DMH and 0.5\% SKTK treated group; D, rectum of DMH and $1.5 \%$ SKTK treated group.

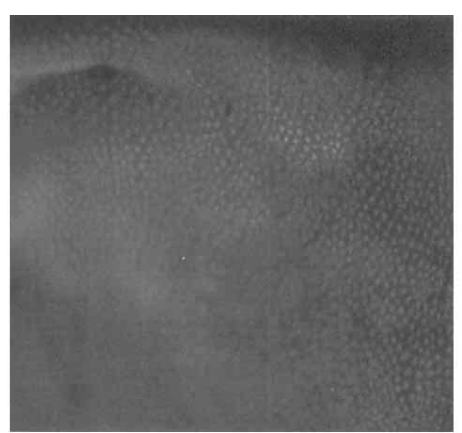

E

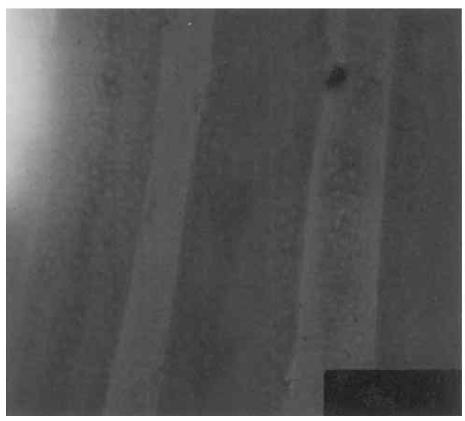

G

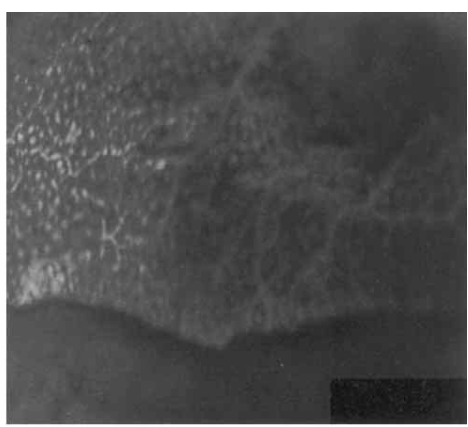

F

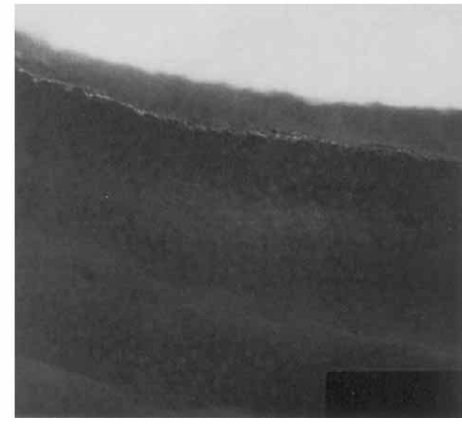

$\mathrm{H}$

Fig. 8. Effect of SKTK on the Formation of Aberrant Foci and Crypts in Murine Colon Induced by 1,2-Dimethylhydrazine

E, sigmoid (S) and descending colon (D) of normal group; F, sigmoid and descending colon of DMH treated group; G, S and D of DMH and $0.5 \%$ SKTK treated group; H, S and $\mathrm{D}$ of DMH and $1.5 \%$ SKTK treated group. 